\title{
SURGICAL TREATMENT OF PULMONARY ASPERGILLOMA: CURRENT OUTCOME
}

Gerard Babatasi, MD, PhD

Massimo Massetti, MD

Alain Chapelier, MD, PhD

Elie Fadel, MD

Paolo Macchiarini, MD, PhD

Andre Khayat, MD

Philippe Dartevelle, MD
Objective: This retrospective study was designed to confirm that aggressive pulmonary resection can provide effective long-term palliation of disease for patients with pulmonary aspergilloma.

Methods and results: From 1959 to 1998,84 patients underwent a total of 90 operations for treatment of pulmonary aspergilloma in the MarieLannelongue Hospital. The mean follow-up period was 9 years, and $83 \%$ of the patients were followed up for 5 years or until death, if the latter occurred earlier. The median age was 44 years. The most common indications were hemoptysis (66\%) and sputum production (15\%). Fifteen patients (18\%) had no symptoms. Tuberculosis and lung abscess were the most common underlying causes of lung disease $(65 \%)$. The procedures were 70 lobar or segmental resections, 8 cavernostomies, and 7 pneumonectomies. Five thoracoplasties were required after lobectomy (3 patients) or pneumonectomy ( 2 patients). The operative mortality rate was $4 \%$. The major complications were bleeding (23 patients), prolonged air leak (31 patients), respiratory failure (10 patients), and empyema (5 patients). The actuarial survival curve showed $84 \%$ survival at 5 years and $74 \%$ survival at 10 years. During the first 2 years, death was related to the surgical procedure and the underlying disease. In contrast, $85 \%$ of the survivors had a good late result.

Conclusion: Lobar resection in both the symptomatic and the asymptomatic patients was conducted in low-risk settings. For patients whose condition is unfit for pulmonary resection, cavernostomy may need to be undertaken despite the high operative risk. The better survival rate in this study may have been due to the selection of patients with better lung function and localized pulmonary disease. (J Thorac Cardiovasc Surg 2000;119:906-12)
Qince the original pathologic description of human Oaspergillosis, by Virchow ${ }^{1}$ in 1856 , the most unusual form of Aspergillus infection recognized is aspergilloma. Hinson and colleagues ${ }^{2}$ have classified pulmonary aspergillosis into allergic, invasive (rarely of surgical concern), and saprophytic infections, which colonize preexisting lung cavities and produce a fungus ball, or aspergilloma. Pulmonary aspergillo-

From Department of Thoracic, Vascular and Heart-Lung Transplantation, Hôpital Marie-Lannelongue, Le PlessisRobinson, and Division of Thoracic and Cardiovascular Surgery, University Hospital, Caen, France.

Received for publication Feb 12, 1999; revisions requested June 11, 1999; revisions received Dec 23, 1999; accepted for publication Dec 27, 1999.

Address for reprints: G. Babatasi, MD, PhD, Division of Thoracic and Cardiovascular Surgery, University Hospital-CHU, 14033Caen, France (E-mail: babatasi-g@chu-caen.fr).

Copyright (C) 2000 by The American Association for Thoracic Surgery

$0022-5223 / 2000 \$ 12.00+0 \quad \mathbf{1 2 / 1 / 1 0 5 2 5 6}$

doi:10.1067/mtc.2000.105256 sis is reported with increasing frequency. Controversy still exists concerning the optimum management of aspergilloma. ${ }^{3}$ With the widespread use of chemotherapy, invasive pulmonary aspergillosis is increasingly reported, occurring in up to $20 \%$ of patients who undergo treatment of acute leukemia. ${ }^{4}$ High morbidity and mortality rates have been reported from surgical series. ${ }^{5,6}$ Some authors have advocated the prophylactic resection of all pulmonary aspergillomas (PA) because of the risk of massive hemoptysis. ${ }^{6-8}$ Other authors recommend surgical treatment only after hemoptysis has occurred. ${ }^{9,10}$ A proportion of the patient population will have advanced chronic lung disease to a degree that eliminates the surgical option. Some authors consider that surgical resection should be avoided because of the high incidence of postoperative complications. ${ }^{11}$ In this study we review the results for all patients who underwent surgical management of PA at the Department of Thoracic Vascular Surgery and Heart-Lung Transplantation at MarieLannelongue Hospital over a 39-year period. 
Table I. Symptoms in 84 patients with PA

\begin{tabular}{lc}
\hline \multicolumn{1}{c}{ Symptom } & $n$ \\
\hline Cough & 13 \\
Hemoptysis & \\
$\quad$ Minor & 54 \\
$\quad$ Major & 2 \\
Fever & 6 \\
Weight loss & 2 \\
Sputum & 13 \\
Chest pain & 2 \\
Asymptomatic & 15 \\
\hline
\end{tabular}

\section{Patients and methods}

Patient population. Eighty-four patients with PA were evaluated and treated at Marie-Lannelongue Hospital between 1959 and 1998. The group consisted of 63 men and 21 women, with an average age of $44 \pm 13$ years.

The records of those 84 patients were reviewed for immunologic risk factors, preoperative signs and symptoms, preoperative chest radiography findings, indications for surgery, surgical procedures performed, postoperative complications, and long-term follow-up status. All the survival probabilities were calculated by the Kaplan-Meier method and plotted at annual intervals, with the day of surgery as the starting time. Operative death and death from all causes were included in the survival statistics. Analysis of the patient population included classic variables (eg: age, symptoms, chest radiograph, computed tomography [CT] scan) and some specific variables, such as respiratory function (percentage vital capacity and forced expiratory volume in 1 second/forced vital capacity) and serodiagnosis (number of precipitations on immunodiffusion tests). Operative death was defined as any death occurring during the first 30-day period or during the initial hospital stay. Bleeding was considered excessive when the sum of preoperative bleeding plus postoperative drainage over the first 24 hours exceeded $1500 \mathrm{~mL}$. Pleural space problems included air leaks prolonged beyond 10 days. The comparisons were conducted with the $\chi^{2}$ test.

Hemoptysis, the most frequent symptom, occurred in 56 patients $(66 \%)$. The degree of hemoptysis was categorized by the amount of blood lost in 24 hours. Hemoptysis was considered massive if the amount was more than $300 \mathrm{~mL}$, severe if between 150 and $300 \mathrm{~mL}$, and moderate if less than $150 \mathrm{~mL}$. Hemoptysis was massive in 11 patients, severe in 19 patients, and moderate in 26 patients. Chronic cough and copious sputum production were the primary symptoms in 13 patients $(15 \% \pm 7 \%)$. Fifteen patients $(18 \% \pm 8 \%)$ were asymptomatic (Table I). Concomitant pulmonary diseases were frequent (Table II). Tuberculosis occurred in 44 patients $(60 \%)$; lung abscess occurred in 11 patients $(13 \%)$; primary chronic obstructive airways disease was present in 5 patients $(6 \%)$, and 4 patients $(4 \%)$ had an underlying hematologic disease, such Hodgkin's lymphoma or acute lymphoblastic leukemia. PA was diagnosed in each patient by one or more methods. The diagnosis was initially sus-
Table II. Underlying lung disorders in 84 patients with PA

\begin{tabular}{lr}
\hline \multicolumn{1}{c}{ Disorder } & $n$ \\
\hline Normal lung & 9 \\
Mycobacterium & 44 \\
Bullae, bleb & 5 \\
Chronic infection & 11 \\
Acute infection (sporotrichosis) & 2 \\
Malignant lesions & 1 \\
Sarcoidosis & 1 \\
\hline
\end{tabular}

Table III. Chest radiograph or CT scan findings and location of PA in 84 patients

\begin{tabular}{lc}
\hline & $n$ \\
\hline Radiologic and CT scan findings & 38 \\
Cavity & 35 \\
Mass & 7 \\
Infiltrate & 4 \\
Emphysema & \\
Location & 36 \\
Right upper lobe & 4 \\
Right middle lobe & 2 \\
Right lower lobe & 24 \\
Left upper lobe & 3 \\
Left lower lobe & 1 \\
Multiple locations & \\
\hline
\end{tabular}

pected in all patients on the basis of the chest radiograph findings. The methods also included bronchoscopy with bronchoalveolar lavage, immunodiffusion tests, and chest CT scans. Sputum or bronchoscopic aspirate cultures were repeatedly positive for Aspergillus fumigatus in 27 patients (32\%). Immunodiffusion tests detected precipitins in 66 patients $(78 \%)$. All the patients in this series had radiographic findings of acute or chronic pulmonary disease with cavities (Table III). Tomography and, more recently, CT scans confirmed the classic picture of an intracavitary mass surrounded by a crescent of air. The cavities were situated in the upper lobes (60 patients) or superior segment of the lower lobe. Cavities were present bilaterally in 2 patients. The maximum diameter varied from 1.5 to $9 \mathrm{~cm}$ (mean, $4 \mathrm{~cm}$ ). The mean ratio of forced expiratory volume to vital capacity was 72 for the lobectomy group, 63 for the pneumonectomy group, and 58 for the cavernostomy group $(P<.01)$. Mean vital capacity (expressed as a percentage of the normal values for the sex, age, and weight) was $90 \pm 12$ for the lobectomy, $65 \pm 17$ for the pneumonectomy, and 56 \pm 16 for the cavernostomy group $(P<.01)$. Preoperative pulmonary function tests showed a mean forced expiratory volume in $1 \mathrm{~second} /$ forced vital capacity of 2.01/2.60.

Operative procedures. The operative procedures, shown in Table IV, included 70 lobar or segmental resections, 7 pneumonectomies, 8 cavernostomies, and 5 thoracoplasties (subsequent to lobar resections or pneumonectomies). 
Table IV. Surgical procedures performed in 84 patients with $P A$

\begin{tabular}{lc}
\hline Procedure & $n$ \\
\hline Initial & \\
Lobectomy & 46 \\
Segmentectomy & 18 \\
Wedge excision & 6 \\
Pneumonectomy & 6 \\
Cavernostomy & 8 \\
Subsequent & \\
Thoracoplasty & 5 \\
Pneumonectomy & 1 \\
\hline
\end{tabular}

Lobectomy and segmentectomy were the most frequent initial procedures for PA. Wedge excision ${ }^{6}$ was required in patients with either small peripheral lesions or poor respiratory functional reserve. Cavernostomy ${ }^{8}$ was performed under local or regional anesthesia through a 5 -cm incision overlying the aspergilloma cavity, guided by echography. The cavity was then incised, and the fungus ball was removed and sent to a pathologist. A short segment of the overlying rib was then excised before the insertion of 2 chest drains. All patients with cavernostomy underwent daily dressing changes and local instillation of amphotericin B. To minimize the risk of postoperative complications, the ipsilateral latissimus dorsi muscle was mobilized from its distal attachment, tunneled, and fixed into the residual cavity (in the presence of the plastic surgeon) in the case of atrophy of the extrathoracic skeletal muscle. This care was continued for 2 to 6 months, followed by closure with a muscle flap in 4 patients. Cavernostomy alone is usually proposed to the most challenging group of patients, including those patients with severe hemoptysis who have had severe lung destruction associated with a prohibitive risk of morbidity (Fig 1). Aspergillus was histologically confirmed on all resected specimens.

Follow-up. A bronchopleural fistula with persistent air space developed in 5 patients (after lobectomy [3 patients] and after pneumonectomy [2 patients]). Thoracoplasty was required in all 5 patients. During follow-up, there were 13 late deaths and 7 deaths from respiratory disease other than aspergilloma ( 3 deaths from acute pneumonia and 4 deaths from chronic respiratory insufficiency). Four patients died of nonrespiratory causes (myocardial infarction, stroke, lung cancer), and 2 patients died of unknown reasons (Table V). No patient died of aspergillosis. One patient had more than 1 aspergilloma. The interval between the first and the second aspergilloma, as asserted by reviewing chest radiographs, was 3 years. The first aspergilloma was resected with lobectomy, followed by thoracoplasty 5 months later, and the second aspergilloma was treated with ipsilateral pneumonectomy. This patient is free of symptoms. Cavernostomy was performed in 8 patients for whom pulmonary resection was considered too dangerous because of their extensive lung disease and poor pulmonary function (mean vital capacity, $56 \pm$

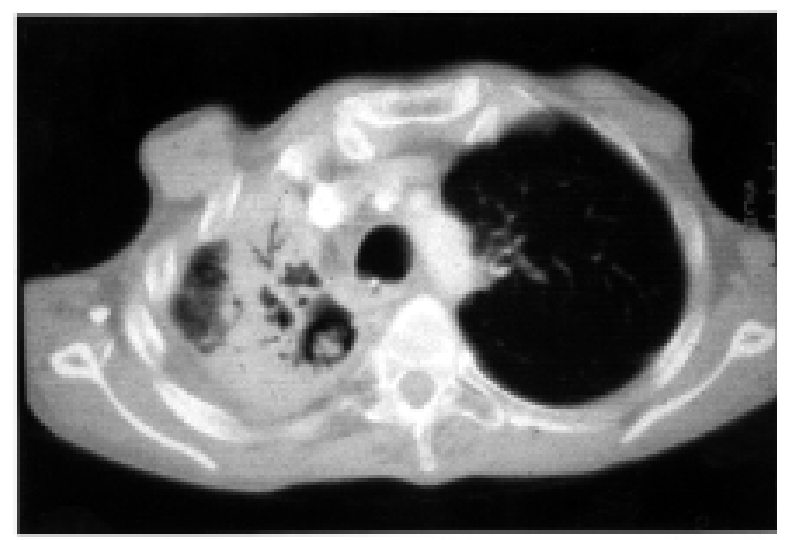

Fig 1. A CT scan of a PA, with surrounding destroyed lung tissue in a patient with previous thoracoplasty.

16 vs $90 \pm 12$ in the lobectomy group; $P<.01$ ). One patient died of pneumonia after cavernostomy. Another patient died of respiratory insufficiency that occurred 5 years after the resection of a chronically suppurating upper lobe. The 6 other patients are currently alive.

\section{Results}

One preoperative death, from massive preoperative bleeding, occurred early in the center's experience (1960), and 3 other deaths occurred after the operation. One patient died of underlying disease (hemopathy) 8 days after lobectomy, and 2 patients' deaths in the postoperative course were related to severe bronchopulmonary infections and respiratory failure (Table V). Fifty-eight patients had 69 nonfatal complications (Table VI). Postoperative hospital stay exceeded 30 days for 16 patients. Major blood loss was noted in 23 patients, and pleural space problems existed in 31 patients. Thoracoplasty was required in 5 patients. Ten patients experienced respiratory distress, 5 of whom needed tracheotomy for long-term respiratory assistance.

The perioperative mortality rate was $4 \%$. The most common preexisting lung lesion was an open-healed tuberculous cavity in 44 patients (52\%). Other common lesions were other forms of upper-lobe fibrosis, sarcoidosis, and allergic bronchopulmonary aspergillosis. Eleven patients had fibrotic or cavitary disease for which no cause was found, and 9 patients had no known previous lung disease. Seventy patients (83\%) were followed up for a minimum of 6 years unless death occurred; 31 patients were followed up for 10 years or more. Three patients were lost to follow-up immediately after discharge, and 5 patients were lost to follow-up after a mean period of 5 years. The actuarial 
survival curve (Fig 2) for 84 patients showed $84 \%$ survival at 5 years, $74 \%$ at 10 years, and $67 \%$ at 15 years.

\section{Comments}

When Aspergillus colonizes a preexisting cavity, the result is a fungus ball or mycetoma that consists of branched, septate hyphae, and blood cells. McPherson ${ }^{12}$ estimated the prevalence of aspergilloma at $0.01 \%$, based on a 10-year survey of chest radiographs in a population of 60,000 patients. A British cooperative study found that patients with a history of tuberculosis and thick-walled lung cavities of more than a 7-year duration had a higher risk of aspergilloma. ${ }^{13}$

From 1959 to 1992 , the most common preexisting condition was tuberculosis. Since that date, the prevalence of PA has slightly decreased. Sixty-three patients underwent operation between 1959 and 1992, whereas only 21 patients underwent operation in the last 6 years. This decrease seems to be related to the regression of the prevalence of sequelae of pulmonary tuberculosis and the extensive use of new antifungal agents. ${ }^{14-16}$

The clinical picture of aspergilloma ranges from an incidental radiologic finding to life-threatening hemoptysis. The survey of the patients spans 39 years. During that time different physicians managed apparently similar cases in different ways. We have confirmed that the upper lobes are the predominant sites of aspergillomas. The size of the aspergillomas was variable and bore no relation to the severity of hemoptysis. Fifteen patients (18\%) were asymptomatic, like those in the series published by Jewkes and colleagues, ${ }^{17}$ in which $22 \%$ of the patients were symptom free. This may be explained by the fact that our series is confined to patients who were admitted to a university hospital and therefore consists of more severely affected patients who were referred by the Department of Chest Medicine for a surgical option. The natural history of aspergilloma is not well documented. Few long-term follow-up reports have been published, and in many of the reports, it is difficult to distinguish between the course of the underlying disease and that of the aspergilloma. ${ }^{18-21}$ A definitive diagnosis of aspergilloma is usually established by the characteristic appearance of a fungus ball on chest radiograph, tomograph, or CT scan (Fig 2). Although CT scan is thought to be more effective in the diagnosis of incipient disease, ${ }^{22}$ we observed no advantage over the more classic tomographs. Isolated growth of Aspergillus from sputum culture is not diagnostic, but positive sputum cultures have repeatedly had more significance. Twenty-seven patients $(32 \%)$ repeatedly had positive sputum cultures for Aspergillus. The serum test for precipitins has been
Table V. Deaths in 84 patients with PA

\begin{tabular}{lc}
\hline \multicolumn{1}{c}{ Causes of death } & $n$ \\
\hline Respiratory disease other than aspergilloma & 7 \\
$\quad$ Acute pneumonia & 3 \\
$\quad$ Chronic respiratory insufficiency & 4 \\
After surgery for aspergilloma & 4 \\
Nonrespiratory causes (eg, myocardial infarction, stroke) & 4 \\
Unknown & 2 \\
Total deaths & 17 \\
\hline
\end{tabular}

found to be both a sensitive and specific method for detecting antibodies to A fumigatus. ${ }^{5}$ Immunodiffusion tests detected precipitins in 66 patients $(78 \%)$. In the event of recent chest radiograph modifications such as infiltration, cavitation, and cyst formation in patients with chronic lung disease, the patients should be screened for Aspergillus infection with the use of immunodiffusion tests. It would appear that the risk of an aspergilloma causing the hematogenous spread of the fungus is negligible. ${ }^{23}$ The definitive diagnosis of aspergilloma is established by demonstrating and culturing the organism from the resected specimen.

Pulmonary aspergillosis, particularly the intracavitary type, can cause sudden and massive hemoptysis. In reported series, ${ }^{5,16,21}$ the incidence of hemoptysis in patients with an aspergilloma has ranged from $50 \%$ to $83 \%$ and is severe or recurrent in $10 \% .^{21}$ Bleeding classically occurs from bronchial arteries and usually stops spontaneously. Several mechanisms for the hemoptysis have been proposed included erosion of the vascular cyst wall by motion of the mycetoma, elaboration of endotoxin by the fungus, and the patient's underlying disease. ${ }^{7}$ Some of these patients experienced massive bleeding; the first hemoptysis is often fatal. The reason for this is unclear. Young and colleagues ${ }^{16}$ postulate that bleeding in these cases may be from intercostal arteries. Extension of the mycotic process with parenchymal destruction at the periphery of the lung invading the adjacent chest wall may lead to erosion of the intercostal arteries. Bleeding from such large arteries is unlikely to stop spontaneously. Neither the size, the complexity of the lesion, the presence of a warning minor hemoptysis, nor the type of underlying disease can predict those patients who will progress to lifethreatening hemoptysis. ${ }^{17}$ Systemic antifungal agents (given orally or intravenously or by inhalation or direct instillation into the cavity), which have been shown to be effective in superficial infections and in some systemic fungal infections, have shown no consistent success in alleviating symptoms or treating the disease process. ${ }^{21}$ Several studies ${ }^{21,24}$ have shown that antifun- 
Table VI. Postoperative outcome and the surgical technique

\begin{tabular}{lcccc}
\hline \multicolumn{1}{c}{ Variable } & $\begin{array}{c}\text { Lobectomy } \\
(n=70)\end{array}$ & $\begin{array}{c}\text { Pneumonectomy } \\
(n=7)\end{array}$ & $\begin{array}{c}\text { Cavernostomy } \\
(n=8)\end{array}$ & $\begin{array}{c}\text { Thoracoplasty } \\
(n=5)\end{array}$ \\
\hline Death & 2 & 1 & 1 & - \\
Prolonged air leak & 27 & - & 4 & - \\
Hemorrhage & 19 & 3 & 1 & 1 \\
Empyema & 3 & 2 & 2 & 5 \\
Respiratory problem & 5 & 2 & 5 & $60.1 \pm 14.1$ \\
Hospital stay $>30 \mathrm{~d}$ & 4 & 2 & $56.3 \pm 16.2$ & $56.8 \pm 1.0$ \\
Vital capacity & $90 \pm 12.2$ & $65 \pm 17.8$ & $58.5 \pm 1.5$ & \\
FEV/VC & $72.6 \pm 1.8$ & $63.5 \pm 2.0$ & & \\
\hline
\end{tabular}

$F E V$, Forced expiratory volume in 1 second $(P<.01)$; $V C$, vital capacity.

*Percentage of the normal values (mean $\pm \mathrm{SD} ; P<.01$ ).

Wedges and segmentectomies included.

gal treatment varies considerably from case to case and does not affect the size of the aspergilloma or death. In our series, 9 patients with PA had been unsuccessfully treated with antifungal therapy and were referred for surgical management. Bronchial artery embolization has been largely unsuccessful because of difficulty in identifying the bleeding artery.

The main goal of the surgical procedure is to resect cavitations near the pulmonary vessels to prevent fatal hemoptysis although limiting the parenchymal resection as much as possible not to impair lung function. There is no general agreement on the management of PA. Gerstl and colleagues ${ }^{25}$ reported successful lobectomy for pulmonary aspergillosis in 1948. Since then, surgical resection has become widely accepted as the treatment of choice for intracavitary mycetoma (fungus balls). Lobectomy is the preferred procedure, but PA have been removed by segmentectomy. Pneumonectomy is required in widespread disease or multiple unilateral aspergilloma and should be performed when there is substantial diffuse infiltration surrounding destroyed lung tissue (ie, from previous tuberculosis) and when there was also an associated fungus ball in one of the involved lobes. ${ }^{20}$ Extension of the aspergilloma and the underlying pulmonary disease should be considered. The high risk of pneumonectomy for aspergilloma is primarily due to the associated technical difficulties that are the result of the often obliterated pleural space and indurated hilar structures. In patients with an invasive or pyogenic component, there exists the possibility of postoperative bronchopleural fistula and empyema. It could be helpful to cover the resected bronchus with a pericardial, pleural, or omental flap. These surgical techniques have been proposed in the last patients who have undergone operations since 1998. The effort always is made to conserve lung tissue because most of these patients have diminished pulmonary reserve.
With the improvement of the surgical techniques (assiduous techniques to reduce both air leaks and postoperative hemorrhage), aggressive surgery ${ }^{6}$ offers, even in asymptomatic patients, 4 potential benefits: prevention of hemoptysis, eradication of the pyogenic component, limitation of the symptoms as the result of invasive aspergillosis or increased growth of the mycetoma, and prolongation of life. Several authors $5,6,22$ insisted that all good-risk patients, even if asymptomatic, should undergo lung resection to avoid the possibility of exsanguinating hemorrhage. However, PA is essentially a benign disease, so the risk of early postoperative death should be completely avoidable. This experience associated with an extremely low morbidity rate has led to our present policy of advocating prophylactic resection whenever a fungus ball is diagnosed if the patient is a suitable candidate for operation. The overall postoperative mortality rate in our series was $4 \%$, similar to that reported by others such as Garvey and colleagues ${ }^{5}(9 \%)$, Massard and colleagues ${ }^{6}(9.5 \%)$, Daly and colleagues ${ }^{10}(5 \%$ in simple aspergilloma, 34\% in complex aspergilloma), and Karas and colleagues ${ }^{11}(13 \%)$. Pleural space problems were the most common postoperative incidents in our series (31 patients; 35\%). In our study until 1992, the measures used to prevent prolonged air leak, particularly after upper lobectomy, consisted in apical pleural tenting. In the last years, with the exception of the application of staple reinforcement materials, including expanding polytetrafluoroethylene sleeves, we used fibrin glue, which has been shown to be an effective sealant for pulmonary parenchymal defects.

We agree with Massard and colleagues ${ }^{6}$ that thoracoplasty may be indicated subsequent to lobectomy. In our series, 5 thoracoplasties were performed as a second choice procedure after lobectomy (3 patients) 


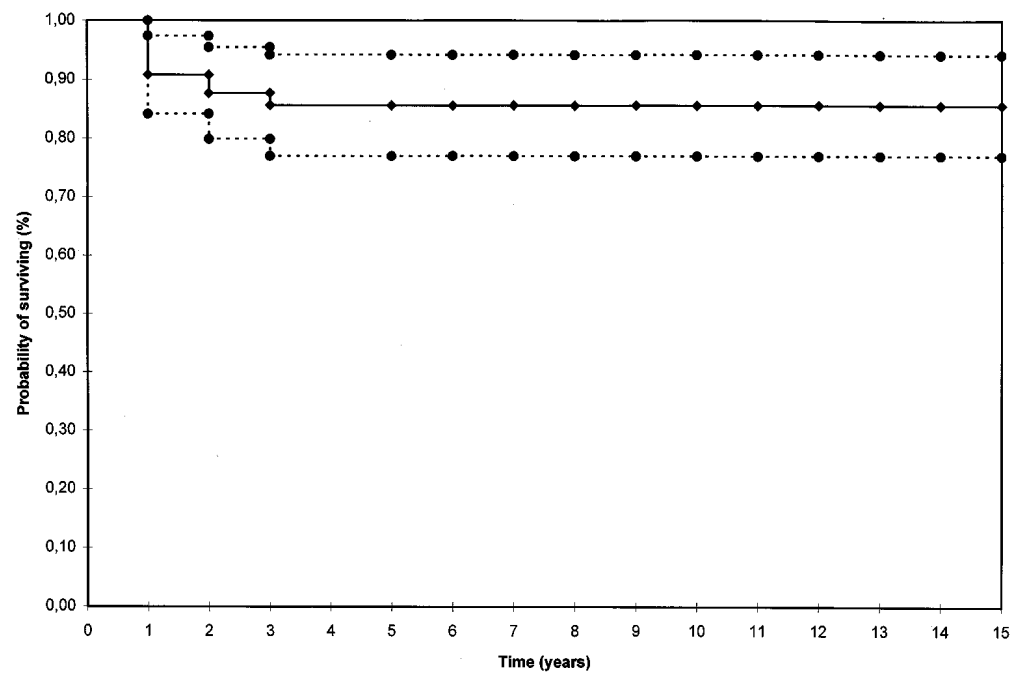

Fig 2. The number of patients surviving beyond 1, 2, 5, and 10 years was 63, 49, 24 and 13, respectively.

or pneumonectomy (2 patients) for bronchopleural fistula or empyema. The outcome after thoracoplasty was good with no deaths, and only 1 patient had respiratory insufficiency. Postoperative bleeding is also a common complication. In the second period (19921998), the incidence of bleeding decreased from $30 \%$ to $7 \%$. This decrease is probably related to the regression of the prevalence of severe long-term sequelae to pulmonary tuberculosis. ${ }^{18}$ There remains a subgroup of patients whose general medical condition or pulmonary function mitigates against resection. To reduce operative complications in patients in poor general condition and if the aspergilloma is located peripherally, an alternative approach consists in cavernostomy and the obliteration of the cavity with intrathoracic transposition of extrathoracic skeletal muscle. This was conducted in 4 of 8 patients who underwent operation, with good long-term results. Further studies are necessary to substantiate these encouraging results. Pulmonary resection should be performed in patients with PA with a low risk and with a strong likelihood of long-term cure. Shirakusa and colleagues ${ }^{22}$ wrote that, because of the saprophytic character of the organism, it is desirable to limit the resection as much as possible, so as not to decrease lung function. Their analysis was based on the age distribution observed in aspergilloma reports from 10 to 20 years previously. ${ }^{5,10}$ In conclusion, in the light of our experience and the recent literature, ${ }^{6,7,14,18}$ we advise pulmonary resection whenever the diagnosis of mycetoma is confirmed and the patient is a suitable candidate for operation. In a few cases, resection may require subsequent thoracoplasty to prevent space problems and the possible development of empyema.

We thank Remy Morello, MD, biostatistician, Department of Biostatistics and Medical Information, for his statistical assistance.

\section{REFERENCES}

1. Virchow R. Beitrage zur Lehre von den beim menschen vorkommenden pflanzlichen parasiten. Virchows Arch [Pathol Anat] 1856;9:557.

2. Hinson KFW, Moon AJ, Plummer NS. Bronchopulmonary aspergillosis: review and report of eight cases. Thorax 1952;7:317-33.

3. Faulkner SL, Vernon R, Brown PP, Fisher RD, Bender HW. Hemoptysis and pulmonary aspergilloma: operative versus nonoperative treatment. Ann Thorac Surg 1978;25:389-92.

4. Degregorio MW, Lee WMF, Linker CA, Jacobs HA, Ries CA. Fungal infections in patients with acute leukemia. Am J Med 1982;73:543-8.

5. Garvey J, Crastnopol P, Weisz D, Khan F. The surgical treatment of pulmonary aspergillomas. J Thorac Cardiovasc Surg 1977;74:542-7.

6. Massard G, Roeslin N, Wihlm JM, Dumont P, Witz JP, Morand G. Pleuropulmonary aspergilloma: clinical spectrum and results of surgical treatment. Ann Thorac Surg 1992;54:1159-64.

7. Solit RW, McKeown JJ, Smullens S, Fraimow W. The surgical implications of intracavitary mycetomas (fungus balls). J Thorac Cardiovasc Surg 1971;62:411-22.

8. Pecora DV, Toll MW. Pulmonary resection for localized aspergillosis. N Engl J Med 1960;263:785-7.

9. Battaglini JW, Murray GF, Keagy BA, Starek PJK, Wilcox BR. Surgical management of symptomatic pulmonary aspergilloma. Ann Thorac Surg 1985;39:512-6. 
10. Daly RC, Pairolero PC, Piehler JM, Trastek VF, Payne WS, Bernatz PE. Pulmonary aspergilloma: results of surgical treatment. J Thorac Cardiovasc Surg 1986;92:981-8.

11. Karas A, Hankins JR, Attar S, Miller JE, MLaughlin JS. Pulmonary aspergilloma: an analysis of 41 patients. Ann Thorac Surg 1976;22:1-7.

12. McPherson P. Pulmonary aspergillosis in Argyll [letter]. Br J Dis Chest 1965;59:148.

13. The Research Committee of the British Tuberculosis Association. Aspergillus in persistent lung cavities after tuberculosis. Tubercle 1968;49:1-4.

14. Temeck BK, Venzon DJ, Moskaluk CA, Pass HI. Thoracotomy for pulmonary mycoses in non-HIV immuno-suppressed patients. Ann Thorac Surg 1994;58:333-8.

15. Bernard A, Caillot D, Casasnovas O, Couaillier JF, Guy H, Favre JP. The value of surgery in the treatment of invasive pulmonary aspergillosis in neutropenic patients. Rev Mal Respir 1998;15:49-55.

16. Young VK, Maghur HA, Luke DA, McGovern EM. Operation for cavitating invasive pulmonary aspergillosis in immunocompromised patients. Ann Thorac Surg 1992;53:621-4.

17. Jewkes J, Kay PH, Paneth M, Citron KM. Pulmonary aspergilloma: analysis of prognosis in relation to haemoptysis and survey of treatment. Thorax 1983;38:572-8.
18. Chatzimichalis A, Massard G, Kessler R, Barsotti P, Claudon B, Ojard-Chillet $\mathrm{J}$, et al. Bronchopulmonary aspergilloma: a reappraisal. Ann Thorac Surg 1998;65:927-96.

19. Salerno CT, Ouyang DW, Pederson TS, Larson DM, Shake JP, Johnson EM, et al. Surgical therapy for pulmonary aspergillosis in immunocompromised patients. Ann Thorac Surg 1998;65:1415-9.

20. Saab SB, Almond C. Surgical aspects of pulmonary aspergillosis. J Thorac Cardiovasc Surg 1974;68:455-60.

21. Rafferty P, Biggs BA, Crompton GK, Grant IWB. What happens to patients with pulmonary aspergilloma? Analysis of 23 cases. Thorax 1983;38:579-83.

22. Shirakusa T, Ueda H, Saito T, Matsuba K, Kouno J, Hirota N. Surgical treatment of pulmonary aspergilloma and Aspergillus empyema. Ann Thorac Surg 1989;48:779-82.

23. Rosenberg RS, Creviston SA, Schonfeld AJ. Invasive aspergillosis complicating resection of a pulmonary aspergilloma in a non-immunosuppressed host. Am Rev Respir Dis 1982;126:1113-5.

24. Borelli D, Bran JL, Fuentes J, Legendre R, Leiderman E, Levine $\mathrm{HB}$, et al. Ketoconazole, an oral antifungal: laboratory and clinical assessment of imidazole drugs. Postgrad Med J 1979;55:657-61.

25. Gerstl B, Weidman WH, Newman AV. Pulmonary aspergillosis: report of two cases. Ann Intern Med 1948;28:662-4.

\section{Availability of Journal back issues}

As a service to our subscribers, copies of back issues of The Journal of Thoracic and Cardiovascular Surgery for the preceding 5 years are maintained and are available for purchase from Mosby until inventory is depleted. The following quantity discounts are available: $25 \%$ off on quantities of 12 to 23, and one third off on quantities of 24 or more. Please write to Mosby, Inc, Subscription Services, 11830 Westline Industrial Drive, St Louis, MO 63146-3318, or call 800-453-4351 or 314-453-4351 for information on availability of particular issues and prices. If unavailable from the publisher, photocopies of complete issues may be purchased from Bell \& Howell Information and Learning, $300 \mathrm{~N}$ Zeeb Rd, Ann Arbor, MI 48106-1346; 734-761-4700 or 800-521-0600. 